\title{
Radio surveys now both deep and wide
}

\author{
Until now, radio astronomers had to choose between sensitivity or field of view. The new Apertif system provides both, \\ enabling studies of low-mass galaxies, galaxy interactions and fast radio bursts, write Betsey Adams and Joeri van Leeuwen.
}

$\int$ eep, wide-field surveys of the radio sky require telescopes able to sample large fields at high sensitivity. Previously, high-angular-resolution radio telescopes such as the Westerbork Synthesis Radio Telescope (WSRT) provided a single beam from a single receiver. Replacing the single receiver with an array of receivers in the focal plane and forming multiple beams provides a wider field of view. Apertif is the new instrument system that upgrades WSRT in order to achieve this capability. Apertif consists of phased array feeds (PAFs) installed on 12 dishes of the existing WSRT infrastructure. The PAF signals are combined in a powerful set of backends to form 40 beams on the sky with an instantaneous bandwidth of 300 $\mathrm{MHz}$ and a total field of view of 9.5 square degrees (Fig. 1). Apertif is developed, built and operated by ASTRON, the Netherlands Institute for Radio Astronomy.

Apertif is the final outcome of a series of ASTRON technological developments to realize a GHz-frequency phased array system. The computing challenge posed by forming 40 beams per PAF has resulted in the UniBoard and UniBoard ${ }^{2}$ platforms ${ }^{1,2}$, which are generalpurpose, fast I/O compute boards powered by field-programmable gate arrays. The UniBoard technology powers the frontend beamformer, correlator and tied-array beamformers. Output from the latter feeds a powerful GPU cluster (named ARTS) that undertakes real-time searches for fast radio bursts (FRBs).

There are three large-scale Apertif surveys planned. Two imaging surveys will provide a $3 \mathrm{D}$ view of the radio Universe. A time-domain survey will detect and localize one FRB for each week of observing time ${ }^{3}$, and characterize $\sim 100$ new pulsars.

The two complementary imaging surveys provide high-resolution (15") images of the radio continuum and neutral hydrogen $(\mathrm{H} \mathrm{I})$ out to a redshift of $z=0.256$, respectively. The shallow survey will cover $>3,000$ square degrees of sky, with a radio continuum sensitivity 30 times that of the NRAO VLA Sky Survey. The medium-deep survey covers a smaller area and returns to the same patch of the sky multiple times to build up sensitivity. The repeated observation cadence also allows the identification of radio transients that vary on timescales of days to months. The radio continuum data will be taken in full polarization to enable the study of cosmic magnetism. The high angular resolution of the neutral hydrogen images ( $>10$ times that of ALFALFA) will enable a wealth of science: resolved $\mathrm{H}$ I kinematics to trace dark matter haloes, a census of the lowest-mass dwarf galaxies to test galaxy formation models, evidence of interaction and environmental processing from disturbed $\mathrm{H}$ i morphology, and more.

The pulsar and FRB search with Apertif exploits the one-dimensional, equidistant WSRT array layout to reach the full, coherent sensitivity over the entire 9.5 square degree field. This field is searched $24 / 7$ in a dedicated survey grid over the entire Northern Hemisphere, and commensal with any imaging observations. The interferometric, real-time system will uniquely provide immediate FRB localization, which is essential for determining FRB hosts and their nature. In parallel, the deep integrations are sensitive to finding elusive intermittent pulsars.

Both the imaging and time-domain surveys benefit from a strong synergy with LOFAR, the Low-Frequency Array, which is also developed, built and operated by ASTRON. The Apertif imaging surveys cover the same sky as the LOFAR Twometre Sky Survey, providing a wide spectral coverage of over a decade in frequency $(120-1,430 \mathrm{MHz})$ to constrain physical parameters and study the origins of the radio continuum emission. Radio counterparts to gravitational wave events with large positional uncertainty can be detected early through Apertif searches, and then studied at LOFAR frequencies. Finally, the real-time ARTS system enables the immediate follow-up and localization by LOFAR of FRBs detected with Apertif.

Apertif is one of many new facilities coming online in preparation for the Square Kilometre Array (SKA). A comparable instrument to Apertif, the Australian SKA Pathfinder (ASKAP) uses independently developed PAF technology. Apertif and ASKAP, as the first interferometers deploying PAFs, are important test cases for future facilities. Their similar performances and complementary coverage of hemispheres make it natural to combine their data. MeerKAT, the SKA pathfinder developed in South Africa, lacks the instantaneous field of view of Apertif but in turn offers exquisite sensitivity. The sensitivity and field of view of the Canadian CHIME telescope are highly

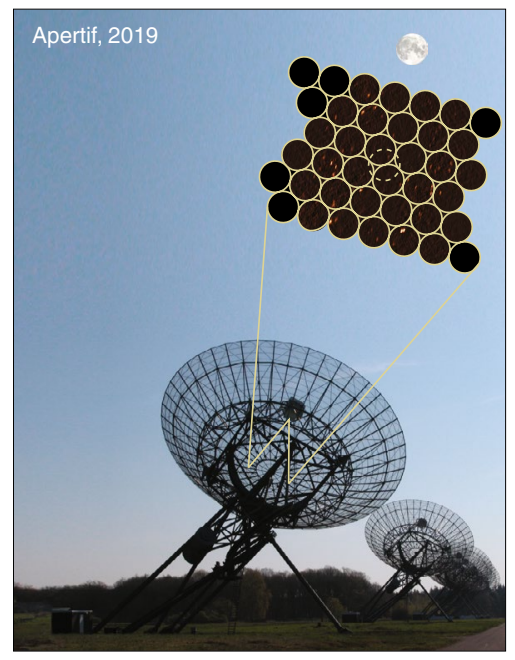

Fig. 1| The improvement in field of view enabled by the 40 beams of Apertif. The previous field of view offered by the WSRT is shown by the dashed circle.

beneficial for FRB detection, albeit with limited localization.

Raw Apertif data will be made public immediately, and higher-level products will be published annually through the Apertif Long-Term Archive (ALTA). Realtime detections of FRBs are communicated as VOEvents ${ }^{4}$ and published to FRBcat.org on detection.

The official opening of Apertif was held on 13 September 2018, which also marked 50 years of WSRT operations. Initial surveys began in January 2019, with full surveys commencing by mid-2019.
Elizabeth A. K. Adams ${ }^{1,2 *}$ and
Joeri van Leeuwen ${ }^{1,3 *}$
${ }^{1}$ ASTRON, the Netherlands Institute for Radio Astronomy, Dwingeloo, The Netherlands. ${ }^{2}$ Kapteyn Astronomical Institute, University of Groningen, Groningen, The Netherlands. ${ }^{3}$ Anton Pannekoek Institute for Astronomy, University of Amsterdam, Amsterdam, The Netherlands.
*e-mail:adams@astron.nl; leeuwen@astron.nl

Published online: 7 February 2019

https://doi.org/10.1038/s41550-019-0692-4

\footnotetext{
References

1. Gunst, A. et al. Exper. Astron. 37, 55-67 (2014).

2. Schoonderbeek, G. W. et al. J. Astron. Instrum. 8, 1950003 (2018)

3. Maan, Y. \& van Leeuwen, J. Preprint at https://arxiv.org/

abs/1709.06104 (2017).

4. Petroff, E. et al. Preprint at https://arxiv.org/abs/1710.08155 (2017).
} 